\title{
Philon's Line Generalized: An Optimization Problem from Geometry ${ }^{1}$
}

\author{
W. W. E. WetTERLING ${ }^{2}$ \\ Communicated by C. G. Broyden
}

\begin{abstract}
Consider in $n$-dimensional Euclidean space the intersection of a convex cone and a hyperplane through a given point. The problem is to minimize the $(n-1)$-volume of this intersection. A geometric interpretation of the first-order optimality condition is given. The special case $n=2$ is known as a characteristic property of Philon's line.
\end{abstract}

Key Words. Philon's line, optimality conditions, convex cones, centroids.

\section{Introduction}

Philon's line appears in a geometric construction for the Delian problem of duplicating a cube. This line has a characteristic extremum property. An analysis of the problem together with historical notes and an extension to non-Euclidean geometry in the plane can be found in a recent article by Coxeter and Van De Craats (Ref. 1). The aim of this paper is to show how that extremum property of a line segment in the plane can be generalized to an extremum property of a hyperplane segment in $n$-dimensional Euclidean space.

\section{Philon's Line}

In the Euclidean plane, consider two straight lines $l_{1}$ and $l_{2}$ intersecting at a point 0 . Let $P \neq 0$ be a point in the plane and $m$ a line through $P$ but

${ }^{1}$ Editorial Note. Because of Professor Wetterling's death on January 21, 1994, this paper was handled by Dr. F. Twilt, Department of Applied Mathematics, University of Twente, Enschede, Netherlands.

${ }^{2}$ Professor, Department of Applied Mathematics, University of Twente, Enschede, Netherlands. 
not through 0 , intersecting $l_{1}$ at $R_{1}$ and $l_{2}$ at $R_{2}$. The perpendicular from 0 on $m$ meets $m$ in $\mathrm{Q}$. The line $m$ is called a Philon line if the distances $\mathrm{R}_{1} \mathrm{P}$ and $Q R_{2}$ are equal ( $P$ and $Q$ between $R_{1}$ and $R_{2}$ or vice versa). If $l_{1}$ and $l_{2}$ are perpendicular, one such line exists for each $P \notin l_{1} \cup l_{2}$, and the equation $x^{3}=a$ can be solved by constructing that line for $\mathrm{P}$ with $\left(l_{1}, l_{2}\right)$ coordinates $(1, a)$. For the Delian problem, choose $a=2$. In Ref. 1 , it is shown that for oblique $l_{1}, l_{2}$ one, two, or three lines $m$ with Philon's property exist, depending on the position of $P$.

For an arbitrary line $m$ as above, let $\Phi(m)$ be the intercept $R_{1} R_{2}$ (distance between $R_{1}$ and $R_{2}$ ). The extremum property mentioned in the introduction states that $m$ is a Philon line if and only if $\Phi$ is stationary at $m$.

\section{Generalization to Higher Dimension}

For a Philon line as in Section 2, the segments $R_{1} R_{2}$ and $P Q$ have the same midpoint. It is this property which allows a generalization. The following notation will be used: $(x, y)$ is the standard inproduct in $R^{n},\|x\|=$ $(x, x)^{1 / 2}$ is the Euclidean norm, and $S=\{u\|\| u \|=1\}$ is the unit sphere.

As generalization of the sector between the lines $l_{1}$ and $l_{2}$ in the preceding section, we consider a closed convex cone $K \subset \mathbb{R}^{n}$ with vertex 0 and nonvoid interior $K^{0}$. We assume $K \backslash\{0\}$ to be contained in a half-space $\{x \mid(a, x)>0\}$, or equivalently, $K \cap(-K)=\{0\}$. In Ref. 2 , a convex cone with this property is called pointed. With our assumptions, the polar cone

$$
K^{p}=\{y \mid(y, x) \leq 0, \text { for all } x \in K\}
$$

is also pointed and has nonvoid interior $\left(K^{p}\right)^{0}$. Since the set $-\left(K^{p}\right)^{0}$ will be needed repeatedly, we introduce the notation

$$
K^{+}=-\left(K^{p}\right)^{0}=\{y \mid(y, x)>0 \text {, for all } x \in K \backslash\{0\}\} .
$$

The line $m$ from Section 2 is generalized to a hyperplane

$$
H(b)=\{x \mid(b, x)=1\} .
$$

Note the one-to-one correspondence between the vectors $b \neq 0$ and the hyperplanes in $\mathbb{R}^{\prime \prime}$ which do not pass through 0 . The intersection $K \cap H(b)$ is compact and nonvoid if and only if $b \in K^{+}$. The length $\Phi(m)$ of the segment $\mathbf{R}_{1} \mathbf{R}_{2}$ is generalized to the $(n-1)$-volume of this intersection,

$$
\Phi(b)=\int_{K \cap H(b)} d \alpha,
$$

with $d \alpha$ the $(n-1)$-dimensional volume element on $H(b)$. 
For $b \in K^{+}$, the projection from the origin $T(u)=u /(b, u)$ defines a bijection $T: K \cap S \rightarrow K \cap H(b)$. For integrable $f$, the substitution rule

$$
\int_{K \cap H(b)} f(x) d \alpha=\|b\| \int_{K \cap S} f(u /(b, u))(b, u)^{-n} d \omega
$$

holds. The easy proof using exterior multiplication of suitable orthonormal vectors and their images under the derivative of $T$ is omitted here. Intuitively, the change in $(n-1)$-volume can be explained as the product of two factors: $1 /(b, u)^{n-1}$, due to the distance factor $1 /(b, u)$ in $T(u)$; and $1 / \cos \delta=$ $\|b\| \cdot\|u\| /(b, u)=\|b\| /(b, u)$, due to the angle $\delta$ between the two normal vectors $u$ of $S$ and $b$ of $H(b)$. The $(n-1)$-volume of $K \cap H(b)$ is now

$$
\Phi(b)=(b, b)^{1 / 2} \int_{K \cap S}(b, u)^{-n} d \omega .
$$

Let a point $p \neq 0$ be given, and consider the optimization problem to minimize $\Phi(b)$ under the constraint $(b, p)=1$. A vector $b$ which satisfies this constraint and the first-order optimality condition with Lagrange multiplier $\lambda$,

$$
D_{b} \Phi=\lambda p,
$$

is called stationary. Note that the assumption $p \neq 0$ is the linear independency constraint qualification from optimization theory. The derivative $D_{b} \Phi$ is easily calculated to be

$$
\begin{aligned}
D_{b} \Phi & =b(b, b)^{-1 / 2} \int_{K \cap S}(b, u)^{-n} d \omega-(b, b)^{1 / 2} n \int_{K \cap S} u(b, u)^{-(n+1)} d \omega \\
& =q \int_{K \cap H(b)} d \alpha-n \int_{K \cap H(b)} x d \alpha,
\end{aligned}
$$

with

$$
q=b /(b, b) \quad \text { and } \quad x=T(u)=u /(b, u) .
$$

Now, $q$ is a multiple of $b$ and satisfies $(b, q)=1$. Hence, $q$ lies on $H(b)$ and is the foot of the perpendicular from the origin on $H(b)$. The integral in the second term is equal to $c \cdot \Phi(b)$, with $c$ the centroid of $K \cap H(b)$. The optimality condition (1) can be written as

$$
(q-n c) \Phi(b)=\lambda p
$$

With

$$
(b, p)=(b, q)=(b, c)=1,
$$


we find that

$$
\lambda=-(n-1) \Phi(b) \text {. }
$$

From our assumption $K^{0} \neq \phi$, it follows that $\Phi(b)>0$. Hence, the optimality condition (1) is equivalent to

$$
c=(1 / n) q+[(n-1) / n] p .
$$

Result 3.1. $\Phi(b)$ is stationary under the constraint $p \in H(b)$ if and only if the centroid $c$ of $K \cap H(b)$ lies on the line through $p$ and the foot $q$ of the perpendicular from the origin on $H(b)$ and divides the line segment between $q$ and $p$ in the ratio $(n-1) / 1$, or in the special case $p=q$, the three points coincide.

For $n=2$, this is the property of Philon's line from Section 2, the points $c, p, q$ being trivially collinear, with $c$ the midpoint of the line segment $p q$.

\section{Open Questions and Remarks}

Several open questions remain: What is the number of stationary points $b$ for a given $p$ ? Which types of stationary points occur (local or global extrema, saddle-points)? What can be said about second-order optimality conditions? A short informal discussion of these questions follows.

(i) A difference between the problems considered in the Sections 2 and 3 must be mentioned. In Section 2 and in Ref. 1 , the lines $l_{1}, l_{2}$ divide $\mathbb{R}^{2}$ into four sectors and the segment $R_{1} R_{2}$ may lie in any of these sectors. The cone $K$ in Section 3 corresponds to only one of the four sectors. The obvious reason lies in the required convexity of $K$ and compactness of $K \cap H(b)$. The results in Ref. 1 imply for the case $n=2$ in our problem that exactly one stationary (minimum) point exists for $p \in K^{0}$ (interior of $K$ ) and that, for $p \notin K$, the number of stationary points is 0,1 , or 2 .

(ii) For a given $p$, write

$$
A(p)=\{b \mid(b, p)=1\} \cap K^{+} .
$$

Suppose that $p \in K^{0}$. Then, the closure $\bar{A}(p)$ is compact, and the $(n-1)$ volume $\Phi(b)$ is finite and continuous for $b \in A(p)$ and tends to infinity when $b$ approaches the relative boundary of $A(p)$. Hence, $\Phi(b)$ has a global minimum on $A(p)$. We have just observed that, for $n=2$, this minimum occurs at the unique stationary $b \in A(p)$. The situation is different for $n \geq 3$. An analysis of the second-order optimality condition for the optimization problem of Section 3 suggests that several stationary points should exist if $K$ is a narrow simplicial cone (spanned by $n$ linearly independent vectors lying 
almost in one hyperplane) and $p \in K^{0}$ close to one of the spanning vectors. It is easily verified that, for the cone $K \subset \mathbb{R}^{3}$ spanned by $(1,-1,-0.2)$, $(1,1,-0.2),(1,0,0.01)$, and the point $p=(1,0,0)$, two local minima and one saddle-point of $\Phi(b)$ exist.

(iii) The ratio $(n-1) / 1$ in Section 3 is no surprise. Considering a special case ( $p$ on the boundary of a simplicial cone $K$ ), we obtain as a corollary of our result the well-known statement: the centroid of an $(n-1)$ simplex divides the line segment between a vertex and the centroid of the opposite facet in that ratio.

(iv) Consider the related problem to minimize the $n$-dimensional volume of the cone segment cut off by a hyperplane through a given point. Using $\Phi(b)$ from Section 3, it is an easy exercise to show that this volume is stationary if and only if $p=c$. This statement holds even more generally without the assumption that $K$ should be a cone; see Ascoli, Ref. 3 .

\section{References}

1. Coxeter, H. S. M., and Van De Craats, J., Philon Limes in Non-Euclidean Planes, Journal of Geometry, Vol. 48, pp. 26-55, 1993.

2. Stoer, J., and Witzgall, C., Convexity and Optimization in Finite Dimensions, Vol. 1, Springer, Berlin, Germany, 1970.

3. Ascolı, G., Sui Baricentri delle Sezioni Piane di un Dominio Spaziale Connesso, Bolletino della Unione Matematica Italiana., Vol. 10, pp. 123-128, 1931. 